\title{
Bilinear Modelling, Control and Stability of Directional Drilling
}

\author{
Isonguyo J. Inyang ${ }^{\mathrm{a}, *}$, James F. Whidborne ${ }^{\mathrm{b}}$ \\ ${ }^{a}$ Advanced Vehicle Engineering Centre, Cranfield University, UK \\ ${ }^{b}$ Centre for Aeronautics, Cranfield University, UK
}

\begin{abstract}
This paper proposes an approach for the attitude control of directional drilling tools for the oil and gas industry. A bilinear model of the directional drilling tool is proposed and it characterises the nonlinear properties of the directional drilling tool more accurately than the existing linear model, hence broadens the range of adequate performance. The proposed bilinear model is used as the basis for the design of a Bilinear Proportional plus Integral (BPI) controller. The stability of the proposed BPI control system is proven using stability notions for LTI and LPV systems. The transient simulation results show that the proposed BPI controller is more effective, robust and stable for the attitude control of the directional drilling tool than the existing PI controller. The proposed BPI controller provides improved invariant azimuth responses and significantly reduces the adverse effects of measurement delays and disturbances with respect to stability and performance of the directional drilling tool.

Keywords: Directional drilling, Attitude control, Bilinear, Time delay, Disturbances, Linear Parameter-Varying (LPV) systems
\end{abstract}

\section{Introduction}

In the oil and gas industry, directional drilling is the practice of creating a geometric wellbore with a directional drilling tool along a predetermined target

\footnotetext{
${ }^{*}$ Corresponding author

Email addresses: i.inyang@cranfield.ac.uk or isonginyang@yahoo.com (Isonguyo J. Inyang), j.f.whidborne@cranfield.ac.uk (James F. Whidborne)
}

Preprint submitted to Journal of ${ }^{A} T_{E} X$ Templates

September 11, 2018 
underneath the Earth's surface for the extraction of petroleum hydrocarbons.

${ }_{5}$ The application of directional drilling creates access to challenging petroleum reservoirs by allowing for the drilling of wells with complex geometries and longer reaching wells (Pedersen et al., 2009). Directional drilling involves attitude, that is, inclination and azimuth angles control of the directional drilling tool. The automatic control of the attitude of the directional drilling tool plays an important role in the maximisation of production as well as reduction of cost per foot of borehole. With the finite reserves of oil and gas resources, reduction in the cost per barrel is desired to cost-effectively match the growing energy requirements for the immediate future.

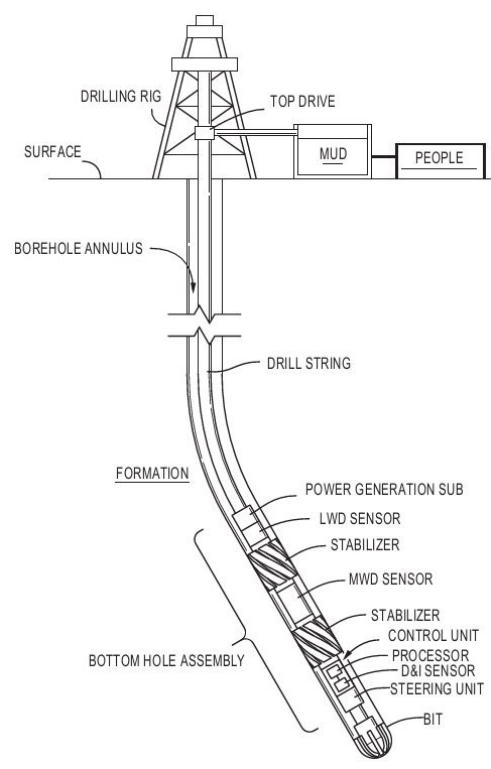

Figure 1: Main components of directional drilling system (Panchal 2013)

A typical directional drilling system with its main components is shown in 15 Fig. 1. During the directional drilling, the drill string, which is the combination of the Bottom-Hole Assembly (BHA) and drill pipe, provides the transmission of torque (from the top drive, located at the surface) and weight to the bit (located downhole). The bit crushes the rock during the propagation of the wellbore. The drilling mud is circulated from the surface to the bit via the drill string to 
lubricate and cool the rock cushing operation and to move the rock-cuttings to the surface via the annulus, that is, the gap between the wellbore and the drill pipe. At the surface, the removal of the cuttings is done, and the recirculation of the drilling fluid is carried out. The BHA comprises of Measurement While Drilling (MWD) subsystem, Logging While Drilling (LWD) subsystem, Power 25 Generation Module (PGM), control unit, Direction and Inclination (D\&I) sensor, steering unit and stabilisers. The LWD subsystem handles the surveys, measurements and assessments of formation properties while a wellbore is propagated, and sometimes shortly after the wellbore propagation, that is, during the periodical stop of the directional drilling operation. The MWD subsystem handles the surveys, measurements and assessments of physical parameters while a wellbore is propagated. The steering assembly (unit) allows for the direction and curvature of the directional drilling tool to be changed as desired during the propagation of the wellbore. Further details of directional drilling system and its operations can be found in Short (1993); Devereux (1999); Baker (1996) 35 and Inyang (2017).

The control unit implements the control algorithms to provide attitude and trajectory control during the directional drilling operations. Note that there is limited communication between the surface and downhole. For example, mud pulse telemetry (Downton, 2012, 2014), which is a technique for the transmis40 sion of commands from the surface to downhole and vice versa, provides about $1 \mathrm{bit} / \mathrm{sec}$ and about $100 \mathrm{bits} / \mathrm{sec}$ communication rates in the less and most favourable of conditions, respectively (Downton, 2012). This technique is significantly affected by measurement noise, the temporal and spatial sampling frequency, and the variability and magnitude of the telemetry delays (Jijón 45 et al. 2010). Hence, the control algorithms must be implemented in the directional drilling tool downhole. The borehole can be several kilometres deep, and the environment is very harsh. The limited power supply and the harsh environment necessitate that the control algorithms must be simple.

To measure the attitude of the directional drilling tool, the D\&I sensor is used, which senses the azimuth angle, $\theta_{\text {azi }}$ and inclination angle, $\theta_{\text {inc }}$ shown in 
Fig. 2. The azimuth angle, $\theta_{\text {azi }} \in\left[0^{\circ}, 360^{\circ}\right)$ is evaluated relative to the Earth's magnetic field projection in the horizontal plane, where $0^{\circ}, 90^{\circ}, 180^{\circ}$ and $270^{\circ}$ correspond to due North, East, South and West, respectively. The inclination angle, $\theta_{\text {inc }} \in\left[0^{\circ}, 180^{\circ}\right]$ is the angular deviation from the vertical with $0^{\circ}, 90^{\circ}$ and $180^{\circ}$ defined downward, horizontal and upward, respectively.

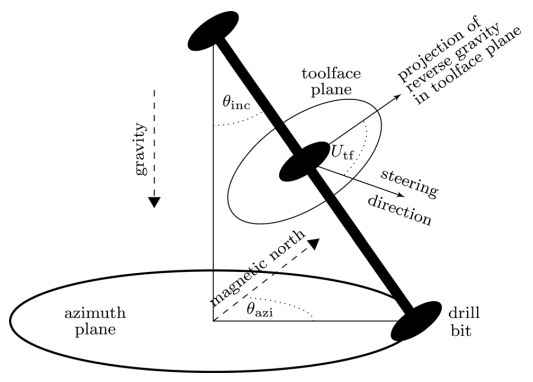

Figure 2: Typical steering and attitude parameters of directional drilling tool

This paper presents the design of a generic tool-independent attitude control algorithm for application to directional drilling tools. The significance of attitude control of directional drilling tools is highlighted in Genevois et al. (2003) and Yonezawa et al. (2002), where control techniques are proposed for Genevois et al. (2003) described the desire for closed-loop "shoot and forget systems" and illustrated the azimuth control to be the major challenge. Most of the attitude control techniques described in literature are elucidated based on specific tool structures. Typical examples are by Yinghui and Yinao (2000),

65 Genevois et al. (2003) and Yonezawa et al. (2002). Another interesting example presented by Kuwana et al. (1994) is an attitude controlling system which uses two-way telemetry communication links with the surface. The steering correction, calculated from the telemetry, is determined and then manually downlinked to the tool. Other control strategies recently developed include a hybrid approach consisting of two levels of automation for trajectory control of the tool (Matheus et al., 2014, 2012), a dynamic state-feedback controller design for 3D directional drilling systems (van de Wouw et al. 2016), a model-based robust 
controller (Kremers et al., 2016), a robust Proportional plus Integral (PI) feedback controller (Panchal et al. 2010), an optimal $H_{\infty}$ controller (Bayliss and

75 Whidborne, 2015) and a linear quadratic Gaussian controller (Bayliss et al. 2015).

When modelling physical systems, the dynamics are often approximated as being linear models obtained by a first-order Taylor series approximation of the nonlinear model at a particular point of operation. It is clear that such linear models might be inaccurate over a wider range of operation; hence, bilinear models have been proposed to more accurately describe the nonlinear systems (see Bruni et al. (1974); Schwarz and Dorissen (1989)). Bilinear models can characterise nonlinear properties over a wider range of operations, hence, broaden the range of adequate performances and they are considered to be significantly advantageous in applications to practical industrial systems (Martineau et al. 2002). In this paper, a bilinear model of the directional drilling tool is developed by applying the Carleman bilinearisation technique (Ghasemi et al. 2014, Krener, 1974, Rugh, 1981). The generalised state space representation of a Multiple-Input Multiple-Output (MIMO) continuous-time bilinear system is expressed as (Kim and Lim, 2003):

$$
\dot{x}=A x+\left(B+\sum_{i=1}^{N} x_{i} M_{i}\right) u
$$

where $A, B$ and $M_{i}$ are constant matrices of suitable dimensions, $u \in \mathbb{R}^{m \times 1}$ denotes the control vector, $x \in \mathbb{R}^{n \times 1}$ represents the vector of state variables and $N$ denotes the number of expansion terms and augmented states.

The PI controller proposed by Panchal et al. (2010) provides good performance for the attitude control of the directional drilling tool but is insufficiently robust to cope with the disturbances and long time delay on the feedback measurements. Furthermore, it is designed at a particular operating point, hence leading to the inconsistency of the azimuth responses for different operating ${ }_{85}$ points. These disturbances are as a result of varying rock formations, a proclivity for the tool to drift horizontally, and to drop towards a vertical orientation due to gravity. While the long time delay arises because the D\&I sensor which 
measures the attitude change is, by necessity, located some distance (sometimes several tens of feet) behind the bit. The time delay increases with the distance of the D\&I sensor from the bit, and inversely proportional to the Rate of Penetration (ROP).

To handle these disturbances, measurement delay and inconsistency of the azimuth responses in the attitude control of the directional drilling tool, this paper, with the extension of some of the works of Panchal et al. (2010) and

${ }_{95}$ Inyang et al. (2016), presents the design of a Bilinear Proportional plus Integral (BPI) controller which automatically holds the attitude of directional drilling tools at the desired attitude, and also significantly reduce the adverse effects of disturbances and measurement delay on stability and performance of directional drilling tools. The BPI controller is considered to provide more consistent azimuth responses over a wider range of directional drilling tool operations. The BPI controller, similar to the Bilinear Proportional-Integral-Derivative (BPID) controllers presented in Martineau et al. 2002, 2004) and Burnham et al. (1999) used in industrial furnace, is relatively simple compared to some of the existing bilinear controllers presented in Lim et al. (2009), Park and Lee (2009), Kim and Lim (2003), Goodhart et al. (1994), Chen et al. (2000), Qian and Zhang (2014), Kelman and Borrelli (2011) and Al-zahrani and Wali (1994) that are used in other different applications. Hence, given the relative simplicity of the BPI controller, it can easily be implemented in directional drilling tools to increase the potential of accessing difficult reservoirs, drilling more effectively and cost-effective field development.

The main contributions of this paper include: (1) The development of a bilinear model of the directional drilling tool which characterises the nonlinear properties of the directional drilling tool more accurately than the existing linear model, hence broadens the range of adequate performance. The proposed bilinear model of the directional tool is used for the design of the BPI controller. (2) The design of a BPI controller for the attitude control of the directional drilling tool. The proposed BPI controller is able to hold the attitude of the directional drilling tool at the desired inclination and azimuth angles and pro- 
vide more consistent azimuth responses over wider range of directional drilling operations. Furthermore, it significantly reduces the adverse effects of time delay on the feedback measurements, disturbances and some other uncertainties (such as drilling cycle and toolface actuator dynamics) in the attitude control of the directional drilling tool.

The remainder of this paper is organised as follows: Section 2 summarises some of the previous models of the directional drilling tool that are related to this paper; and also presents the development and the accuracy of a bilinear model of the directional drilling tool. Section 3 presents the design of a BPI controller for the attitude control of the directional drilling tool. In Section 4 the stability of the proposed BPI control system is proven using stability notions for Linear Time Invariant (LTI) and Linear Parameter-Varying (LPV) systems. Section 5 presents transient results based on Low-Fidelity Model (LFM) and High-Fidelity Model (HFM) simulations.

\section{Models of Directional Drilling Tool}

\subsection{Previous Models}

The directional drilling tool model proposed by Panchal et al. (2010) is derived from kinematic considerations and does not take into consideration the torsional and lateral dynamics of the drillstem and BHA. The model proposed by Panchal et al. (2010) is illustrated in Fig. 2 and it is given as:

$$
\begin{aligned}
& \dot{\theta}_{\text {inc }}=V_{\text {rop }}\left(U_{\mathrm{dls}} \cos U_{\mathrm{tf}}-V_{\mathrm{dr}}\right) \\
& \dot{\theta}_{\mathrm{azi}}=\frac{V_{\mathrm{rop}}}{\sin \theta_{\mathrm{inc}}}\left(U_{\mathrm{dls}} \sin U_{\mathrm{tf}}-V_{\mathrm{tr}}\right)
\end{aligned}
$$

where $V_{\text {rop }}$ denotes the rate of penetration, a time-varying parameter, $V_{\mathrm{tr}}$ denotes the turn rate bias disturbance, $V_{\mathrm{dr}}$ denotes the drop rate disturbance $\left(V_{\mathrm{dr}}=\alpha \sin \theta_{\mathrm{inc}}\right), \alpha$ is a constant, $U_{\mathrm{dls}}$ denotes the curvature, also known as "dogleg severity" which is the product of duty cycle and $K_{\mathrm{dls}}, K_{\mathrm{dls}}$ denotes the open-loop curvature capability of the tool, $U_{\mathrm{tf}}$ denotes the toolface angle control input, $\theta_{\text {azi }}$ denotes the azimuth angle and $\theta_{\text {inc }}$ denotes the inclination 
angle. Note that in (3) there is a singularity when $\theta_{\text {inc }}=0^{\circ}$, hence, the model, (2) and (3) is restricted to attitudes such that $\theta_{\text {inc }}$ is not close to $0^{\circ}$.

The model, (2) and (3) has also been used in Matheus et al. (2014, 2012) (see also Cockburn et al. (2011); Matheus and Naganathan (2010) ) where field tested results are presented that show the fidelity of the model with actual practice.

The model, (2) and (3) is partially linearised and decoupled as follows (Panchal et al. 2010):

$$
\begin{aligned}
& \dot{\theta}_{\mathrm{inc}}=V_{\mathrm{rop}} K_{\mathrm{dls}} U_{\mathrm{inc}} \\
& \dot{\theta}_{\mathrm{azi}}=\frac{V_{\text {rop }}}{\sin \theta_{\mathrm{inc}}} K_{\mathrm{dls}} U_{\mathrm{azi}}
\end{aligned}
$$

where $U_{\text {inc }}$ and $U_{\text {azi }}$ represent the virtual controls for the inclination and azimuth, respectively, and the transformation is given as: $U_{\mathrm{tf}}=\operatorname{ATAN2}\left(U_{\mathrm{azi}}, U_{\mathrm{inc}}\right)$ and $U_{\mathrm{dls}}=K_{\mathrm{dls}} \sqrt{\left(U_{\mathrm{azi}}\right)^{2}+\left(U_{\mathrm{inc}}\right)^{2}}$. With the linearisation of (4) and (5) about a nominal inclination angle, $\hat{\theta}_{\text {inc }}$, a linearised model of the directional drilling tool is obtained as follows (Panchal et al., 2010):

$$
\begin{aligned}
& \dot{x}_{\mathrm{inc}}=a u_{\mathrm{inc}} \\
& \dot{x}_{\mathrm{azi}}=c x_{\mathrm{inc}}+b u_{\mathrm{azi}}
\end{aligned}
$$

where $c=-b \cot \hat{\theta}_{\mathrm{inc}}, b=a \csc \hat{\theta}_{\mathrm{inc}}$ and $a=V_{\mathrm{rop}} K_{\mathrm{dls}}$. Based on (6) and (7), Panchal et al. (2010) designed a PI controller for the attitude control of the directional drilling tool.

\subsection{Proposed Bilinear Model}

In this subsection, the Carleman bilinearisation technique (Ghasemi et al. 2014 Krener, 1974 Rugh, 1981) is applied to the partially linearised and decoupled system, (4) and (5), to obtain a bilinear model of the directional drilling tool. Equations (4) and (5) are rewritten as:

$$
\begin{aligned}
& \dot{\theta}_{\mathrm{inc}}=a U_{\mathrm{inc}} \\
& \dot{\theta}_{\mathrm{azi}}=\frac{a}{\sin \theta_{\mathrm{inc}}} U_{\mathrm{azi}}
\end{aligned}
$$


where $a=V_{\text {rop }} K_{\text {dls }}$. Defining an augmented state vector for the Carleman bilinearisation as:

$$
x^{\otimes}=\left[x_{1}, x_{1}^{(2)}, x_{1}^{(3)}, x_{1}^{(4)}, \ldots, x_{1}^{(N)}, x_{2}\right]^{T}
$$

where $x_{1}=\theta_{\text {inc }}, x_{2}=\theta_{\text {azi }}$ and $\dot{x}_{1}^{(i)}=\frac{d}{d t}\left[\left(x_{1}\right)^{i}\right]=i \dot{x}_{1} x_{1}^{(i-1)}$ leads to an extended bilinear state space system:

$$
\begin{aligned}
& \dot{x}_{1}=a U_{\mathrm{inc}} \\
& \dot{x}_{2}=a U_{\mathrm{azi}} \csc \left(x_{1}\right)=a U_{\mathrm{azi}} \sec (\beta)=a U_{\mathrm{azi}} \sum_{i=1}^{\infty} b_{i} x_{1}^{(i)}
\end{aligned}
$$

where $\beta=\pi / 2-\theta_{\text {inc }}$ and $b_{i}$ are the coefficients of the Taylor series expansion of $\sec (\beta)$. The expansion of $\sec (\beta)$ is used instead of $\csc \left(x_{1}\right)$ so that the required powers of $x_{1}^{(i)}$ can be obtained from the $\theta_{\text {inc }}$ state equation. The Taylor series expansion of $\sec (\beta)$ for $-\frac{\pi}{2}<\beta<\frac{\pi}{2}$ is obtained from

$$
\sec (\beta)=1+\frac{1}{2} \beta^{2}+\frac{5}{24} \beta^{4}+\frac{61}{720} \beta^{6}+\cdots+\frac{(-1)^{n} E_{2 n}(\beta)^{2 n}}{(2 n) !}+\ldots
$$

where $E_{2 n}$ is the Euler number (Rade and Westergren, 1999, pp 81-99).

In practice, the number of terms in the series expansion and the number of augmented states is truncated to a finite value $N$ (where $N$ is equal to the number of expansion terms and augmented states) to an arbitrary degree of accuracy. Expanding (12), then (11) and 12 gives the bilinear system:

$$
\begin{aligned}
\dot{x}_{1} & =a U_{\mathrm{inc}} \\
\dot{x}_{1}^{(2)} & =2 x_{1} \dot{x}_{1}=2 a x_{1} U_{\mathrm{inc}} \\
\dot{x}_{1}^{(3)} & =3 x_{1}^{(2)} \dot{x}_{1}=3 a x_{1}^{(2)} U_{\mathrm{inc}} \\
\vdots & \quad \vdots \\
\dot{x}_{1}^{(N)} & =N x_{1}^{(N-1)} \dot{x}_{1}=N a x_{1}^{(N-1)} U_{\mathrm{inc}} \\
\dot{x}_{2} & =a U_{\mathrm{azi}} \sum_{i=1}^{N} b_{i} x_{1}^{(i)}
\end{aligned}
$$

which is in the form of (1); where $A=[], u=\left[U_{\mathrm{inc}}, U_{\mathrm{azi}}\right]^{T}$ and $x=x^{\otimes}$. For 
example, where $N=3$, the following is obtained:

$$
\begin{aligned}
{\left[\begin{array}{c}
x_{1} \\
x_{1}^{(2)} \\
x_{1}^{(3)} \\
x_{2}
\end{array}\right]=} & {\left[\begin{array}{cc}
0 & 0 \\
2 a & 0 \\
0 & 0 \\
0 & a b_{1}
\end{array}\right]\left[\begin{array}{l}
U_{\mathrm{inc}} \\
U_{\mathrm{azi}}
\end{array}\right] x_{1}+\left[\begin{array}{cc}
0 & 0 \\
0 & 0 \\
3 a & 0 \\
0 & a b_{2}
\end{array}\right]\left[\begin{array}{l}
U_{\mathrm{inc}} \\
U_{\mathrm{azi}}
\end{array}\right] x_{1}^{(2)} } \\
& +\left[\begin{array}{cc}
0 & 0 \\
0 & 0 \\
0 & 0 \\
0 & a b_{3}
\end{array}\right]\left[\begin{array}{l}
U_{\mathrm{inc}} \\
U_{\mathrm{azi}}
\end{array}\right] x_{1}^{(3)}+\left[\begin{array}{cc}
0 & 0 \\
0 & 0 \\
0 & 0 \\
0 & 0
\end{array}\right]\left[\begin{array}{l}
U_{\mathrm{inc}} \\
U_{\mathrm{azi}}
\end{array}\right]
\end{aligned}
$$

The accuracy of the proposed bilinear model of the directional drilling tool is analysed in the remaining part of this section. Furthermore, the proposed bilinear model is used as the basis for the BPI controller design as analysed in Section 3

\subsubsection{Bilinear Model Accuracy}

To demonstrate the accuracy of the proposed bilinear model of the directional drilling tool, the transient azimuth responses for the open-loop systems using the proposed bilinear model for $N=3,4$ and 10 are analysed and compared with those from the nonlinear model, (2) and (3) (with $V_{\mathrm{dr}}$ and $V_{\mathrm{tr}}$ ignored) and the linear model, (6) and (7) proposed by Panchal et al. (2010). It is expected that the inclination responses of the models are identical because there is no nonlinearity in the inclination open-loops of the models. The parameters used for the models comparison simulation are given in Table 1 .

Table 1: Simulation Parameters for Models Comparison

\begin{tabular}{|l|c|c|c|}
\hline Parameter & Value & Parameter & Value \\
\hline$\theta_{\text {azi }}$ & $2.5 \mathrm{rad}\left(143.24^{\circ}\right)$ & $V_{\text {rop }}$ & $100 \mathrm{ft} / \mathrm{hr}(0.5079 \mathrm{~m} / \mathrm{min})$ \\
\hline$N$ & $3,4,10$ & $K_{\text {dls }}$ & $10^{\circ} / 100 \mathrm{ft}(5.7264 \mathrm{e}-3 \mathrm{rad} / \mathrm{m})$ \\
\hline
\end{tabular}




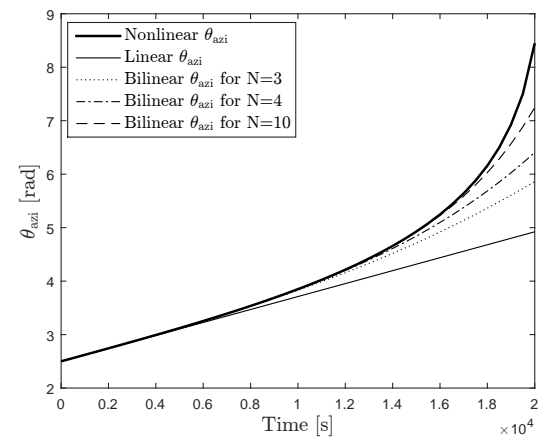

Figure 3: Comparison of azimuth responses of nonlinear, linear and bilinear for $N=3,4$ and 10 models

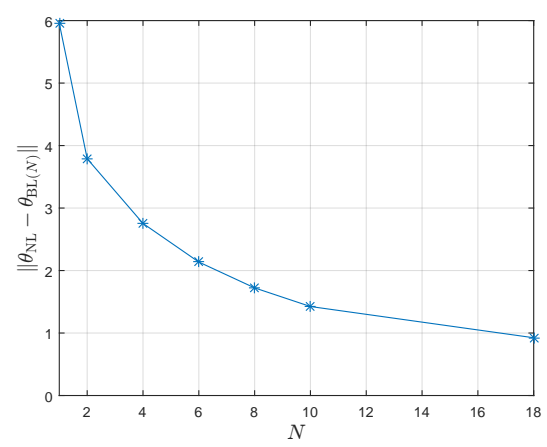

Figure 4: Relationship between the accuracy of bilinear azimuth responses and $N$

Based on the comparison shown in Fig. 3, the azimuth responses of the proposed bilinear model for $N=3,4$ and 10, where the accuracy of the proposed bilinear model improves as $N$ increases, converge more closely to the azimuth response of the nonlinear model than that of the linear model. Hence, the proposed bilinear model characterises the nonlinear model more accurately than the linear model proposed by Panchal et al. (2010), thereby broadening the range of adequate performances, and it is considered to be significantly advantageous in applications to directional drilling operations.

Furthermore, to demonstrate that the accuracy of the proposed bilinear model increases with $N$, the norm error of the nonlinear and bilinear azimuth responses given by $\left\|\theta_{\mathrm{NL}}-\theta_{\mathrm{BL}(N)}\right\|$ as a function of $N$ is shown in Fig. 4 
where $\theta_{\mathrm{NL}}$ is the nonlinear azimuth response and $\theta_{\mathrm{BL}(N)}$ is the bilinear azimuth responses for $N=1,2,4,6,8,10$ and 18. Based on Fig. 4, it is analysed that $\left\|\theta_{\mathrm{NL}}-\theta_{\mathrm{BL}(N)}\right\|$ decreases as $N$ increases. Hence, the accuracy of the proposed bilinear model improves as $N$ increases.

\section{BPI Controller Design}

The proposed BPI controller design scheme, shown in Fig. 5, is based on the works of Martineau et al. (2002, 2004) and Burnham et al. (1999), and it is a combination of a bilinear compensator and a standard linear PI controller. The proposed bilinear compensator is only incorporated in the azimuth feedback loop to account for the nonlinear $1 / \sin \theta_{\text {inc }}$ term in (3).

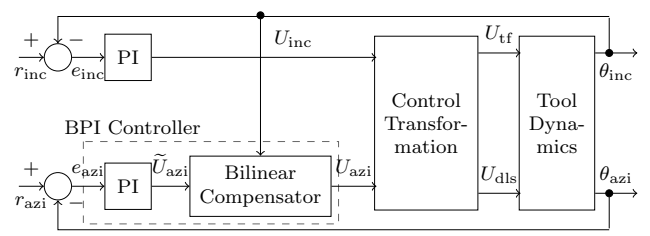

Figure 5: BPI control scheme

\subsection{PI Controller}

The PI control for the inclination and azimuth control channels are as follows, respectively:

$$
\begin{aligned}
& U_{\mathrm{inc}}=k_{\mathrm{pi}} e_{\mathrm{inc}}+k_{\mathrm{ii}} \int_{0}^{t} e_{\mathrm{inc}} d t \\
& \widetilde{U}_{\mathrm{azi}}=k_{\mathrm{pa}} e_{\mathrm{azi}}+k_{\mathrm{ia}} \int_{0}^{t} e_{\mathrm{azi}} d t
\end{aligned}
$$

where $\widetilde{U}_{\text {azi }}$ is the control input to the proposed bilinear compensator; $e_{\mathrm{inc}}=$ $r_{\text {inc }}-\theta_{\text {inc }}$ and $e_{\text {azi }}=r_{\text {azi }}-\theta_{\text {azi }} ; r_{\text {inc }}$ and $r_{\text {azi }}$ are the nominal operating points for inclination and azimuth, respectively; $k_{\mathrm{pi}}$ and $k_{\mathrm{ii}}$ are the proportional and integral gains in the inclination feedback loop, respectively; $k_{\mathrm{pa}}$ and $k_{\mathrm{ia}}$ are the proportional and integral gains in the azimuth feedback loop, respectively. 
The gains for the PI controllers in the inclination and azimuth feedback loops are manually tuned to achieve desired performance in terms of zero steady state error, fast settling time and minimal overshoot, with practical engineering considerations.

\subsection{Bilinear Compensator}

Assuming that in Fig. 5. $U_{\text {azi }}=\widetilde{U}_{\text {azi }}$, then only the PI controller is incorporated (see (17)), and the azimuth feedback loop is expressed based on 12 as follows:

$$
\dot{\theta}_{\mathrm{azi}}=a \sum_{i=1}^{\infty} b_{i} x_{1}^{(i)}\left(k_{\mathrm{pa}} e_{\mathrm{azi}}+k_{\mathrm{ia}} \int_{0}^{t} e_{\mathrm{azi}} d t\right)
$$

Based on (13) and 14, (18) can further be expressed as:

$$
\begin{aligned}
\dot{\theta}_{\mathrm{azi}}= & a\left(1+\frac{1}{2} \beta^{2}+\frac{5}{24} \beta^{4}+\frac{61}{720} \beta^{6}+\cdots+\frac{(-1)^{n} E_{2 n}(\beta)^{2 n}}{(2 n) !}\right. \\
& +\ldots)\left(k_{\mathrm{pa}} e_{\mathrm{azi}}+k_{\mathrm{ia}} \int_{0}^{t} e_{\mathrm{azi}} d t\right)
\end{aligned}
$$

To account for the nonlinearity in 19 , a bilinear compensator is proposed for the azimuth feedback loop which is given as:

$$
\frac{U_{\mathrm{azi}}}{\widetilde{U}_{\mathrm{azi}}}=\frac{1}{1+\frac{1}{2} \beta^{2}+\frac{5}{24} \beta^{4}+\frac{61}{720} \beta^{6}+\cdots+\frac{(-1)^{n} E_{2 n}(\beta)^{2 n}}{(2 n) !}+\ldots}
$$

Clearly, this has an infinite number of terms, hence, for practical implementation, 200 is truncated at $\beta^{4}$, and it is given as:

$$
\frac{U_{\mathrm{azi}}}{\widetilde{U}_{\mathrm{azi}}}=\frac{1}{1+\frac{1}{2} \beta^{2}+\frac{5}{24} \beta^{4}}
$$

Note that, the simulation results of 20 truncated at degrees higher than $\beta^{4}$, have no significant improvement compared to those of (21).

The proposed bilinear compensator, 21) approximately cancels out the nonlinear $1 / \sin \theta_{\text {inc }}$ term in (3), thereby enhancing the performance of the PI controller. The proposed bilinear compensator, 21), in combination with the PI controller, facilitates the ensuing BPI controller to sustain a required degree of control throughout a broader scope of operation about the tuning point compared with that obtained with the PI controller proposed by Panchal et al. 
(2010). Hence, the proposed BPI controller design provides improved invariant clear that the stability of the proposed BPI control system can be analysed by considering the stability of the two feedback loops (inclination and azimuth) separately. With reference to Fig. 6, and based on (8) and (9), $f_{\text {azi }}\left(U_{\text {inc }}\right)=$ $a U_{\mathrm{inc}}=V_{\mathrm{rop}} K_{\mathrm{dls}} U_{\mathrm{inc}}$ and $f_{\mathrm{azi}}\left(U_{\mathrm{azi}}, \beta\right)=a U_{\mathrm{azi}} / \cos \beta(t)($ recall, $\beta(t)=\pi / 2-$ $\left.\theta_{\text {inc }}(t)\right)$, and $K_{\mathrm{b}}\left(\widetilde{U}_{\mathrm{azi}}, \beta\right)$ is given in $(21)$.

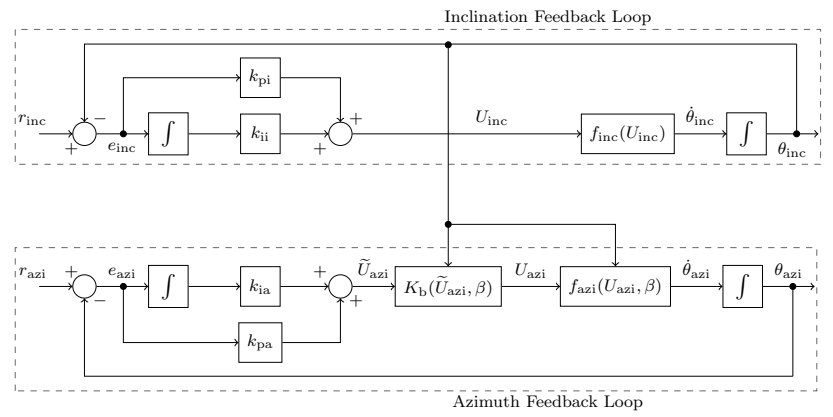

Figure 6: BPI control system
With the introduction of an augmented state, $e_{\mathrm{ai}}$, representing the accumulated inclination error, and based on Fig. 7, the inclination feedback loop with 
the PI controller is written as:

$$
\begin{aligned}
\dot{e}_{\mathrm{ai}}(t) & =-\theta_{\mathrm{inc}}(t) \\
\dot{\theta}_{\mathrm{inc}}(t) & =\left(k_{\mathrm{ii}} e_{\mathrm{ai}}(t)-k_{\mathrm{pi}} \theta_{\mathrm{inc}}(t)\right) a
\end{aligned}
$$

where $e_{\mathrm{ai}}(t), \theta_{\text {inc }}(t) \in \mathbb{R}^{n}$ and $t \in \mathbb{R}^{+}$.

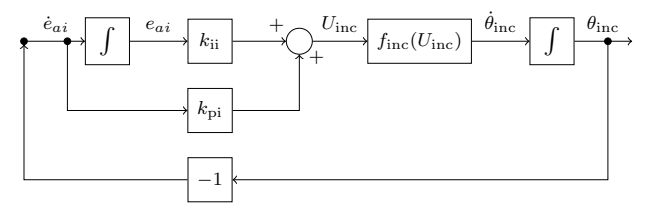

Figure 7: Inclination feedback loop with PI controller

Note that (22) is an LTI system and it can be put in the form of:

$$
\begin{aligned}
& \dot{x}(t)=A x(t), \quad t \geq 0 \\
& x(0)=x_{0}
\end{aligned}
$$

where $x \in \mathbb{R}^{n}$ denotes the system state, $A \in \mathbb{R}^{n \times n}$ denotes system matrix, with $x(t)=\left[e_{\text {ai }}(t), \theta_{\text {inc }}(t)\right]^{T}$ and

$$
A=\left[\begin{array}{cc}
0 & -1 \\
k_{\mathrm{ii}} a & -k_{\mathrm{pi}} a
\end{array}\right]
$$

Clearly, the eigenvalues of $A$ are left-half-plane for all positive $a, k_{\mathrm{ii}}$ and $k_{\mathrm{pi}}$; hence the inclination feedback loop with the PI controller given in 24 is exponentially stable.

The remaining part of this section focuses on the stability analysis of the proposed BPI controller which is incorporated in the azimuth feedback loop (see Fig. 8). With the introduction of an augmented state, $e_{\text {aa }}$, representing the accumulated azimuth error, and based on Fig. 8 , the azimuth feedback loop with the proposed BPI controller is written as:

$$
\begin{aligned}
\dot{e}_{\mathrm{aa}}(t) & =-\theta_{\mathrm{azi}}(t) \\
\dot{\theta}_{\mathrm{azi}}(t) & =\left(k_{\mathrm{ia}} e_{\mathrm{aa}}(t)-k_{\mathrm{pa}} \theta_{\mathrm{azi}}(t)\right) a f(\beta(t))
\end{aligned}
$$


where $e_{\mathrm{a}}(t), \theta_{\text {azi }}(t) \in \mathbb{R}^{n} ; t \in \mathbb{R}^{+}$,

$$
f(\beta(t))=\frac{1}{\cos \beta(t)\left(1+\frac{1}{2} \beta^{2}(t)+\frac{5}{24} \beta^{4}(t)\right)}
$$

$\beta(t) \in[\beta, \bar{\beta}] \operatorname{rad}, \beta$ and $\bar{\beta}$ are the minimum and maximum values of $\beta(t)$, respectively.

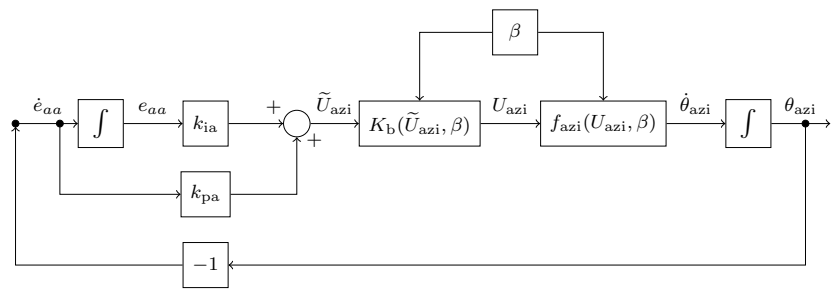

Figure 8: Azimuth feedback loop with BPI controller

Note that 25) is an LPV system and it is rewritten in a generic LPV system form (Briat, 2015, p. 10):

$$
\begin{aligned}
& \dot{x}(t)=A(\rho(t)) x(t), \quad t \geq 0 \\
& x(0)=x_{0}
\end{aligned}
$$

with: $x(t)=\left[e_{\mathrm{aa}}(t), \theta_{\mathrm{azi}}(t)\right]^{T}, \rho(t)=\beta(t), A(\rho(t))=A(\beta(t))$ and

$$
A(\beta(t))=\left[\begin{array}{cc}
0 & -1 \\
a_{1} f(\beta(t)) & -a_{2} f(\beta(t))
\end{array}\right]
$$

where $\rho: \mathbb{R}_{\geq 0} \rightarrow \Delta_{\rho}, \Delta_{\rho}$ denotes the set of values of $\rho, a_{1}=k_{\mathrm{ia}} a$ and $a_{2}=k_{\mathrm{pa}} a$.

Furthermore, 28) is rewritten in a polytopic LPV system form Briat, 2015. p. 56):

$$
\begin{aligned}
& \dot{x}(t)=A(\lambda(t)) x(t), \quad t \geq 0 \\
& x(0)=x_{0}
\end{aligned}
$$


with:

$$
\begin{aligned}
& A(\beta(t))=\lambda_{1}(t) A_{1}+\lambda_{2}(t) A_{2} \\
& \lambda_{1}(t)=\frac{p(t)-\underline{p}}{\bar{p}-\underline{p}}, \lambda_{2}(t)=\frac{\bar{p}-p(t)}{\bar{p}-\underline{p}}, A_{1}=\left[\begin{array}{cc}
0 & -1 \\
a_{1} \underline{p} & -a_{2} \underline{p}
\end{array}\right], A_{2}=\left[\begin{array}{cc}
0 & -1 \\
a_{1} \bar{p} & -a_{2} \bar{p}
\end{array}\right]
\end{aligned}
$$

where $p(t)=f(\beta(t)), p(t) \in[\underline{p}, \bar{p}], \underline{p}$ and $\bar{p}$ are the minimum and maximum values of $p(t)$, respectively, $A(\lambda(t))=\sum_{i=1}^{M} \lambda_{i}(t) A_{i}, A_{i} \in \mathbb{R}^{n \times n}, \lambda(t) \in \Lambda_{M}$, ${ }_{230} \Lambda_{M}=\operatorname{co}\{\mathbf{V}\}, \mathbf{V}$ denotes a set of vertices, $v_{i}$, that is, $\mathbf{V}:=\left\{v_{1}, \ldots, v_{M}\right\}$, $\mathbf{V}=\operatorname{vert}\left\{\Lambda_{M}\right\}$ and $\Lambda_{M}$ denotes a $M$-unit simplex which is defined as: $\Lambda_{M}:=$ $\left\{\lambda \in \mathbb{R}_{\geq 0}^{n}: \sum_{i=1}^{M} \lambda_{i}=1\right\}$.

Based on the fact that 28$)$ is affinely dependent on $p(t),(28)$ is rewritten in an affine parameter-dependent system form (Briat, 2015, p. 10); (Gu et al. 2013 p. 223); (Gahinet et al., 1994, 1996):

$$
\begin{aligned}
& \dot{x}(t)=\left(A_{f 0}+\rho_{1}(t) A_{f 1}+\rho_{2}(t) A_{f 2}+\cdots+\rho_{n}(t) A_{f n}\right) x(t), \quad t \geq 0 \\
& x(0)=x_{0}
\end{aligned}
$$

as:

$$
\dot{x}(t)=\left(A_{f 0}+p(t) A_{f 1}\right) x(t)
$$

where

$$
A_{f 0}=\left[\begin{array}{cc}
0 & -1 \\
0 & 0
\end{array}\right], \quad A_{f 1}=\left[\begin{array}{cc}
0 & 0 \\
a_{1} & -a_{2}
\end{array}\right]
$$

$A_{f 0}, A_{f 1}, A_{f 2}, \ldots, A_{f n}$ are known fixed matrices and $\rho_{1}(t), \rho_{2}(t), \ldots, \rho_{n}(t)$ are time-varying parameters.

Note that in (26) when $\beta(t)=\pi / 2 \mathrm{rad}, f(\beta(t))=\infty$ which clearly leads to instability. To analyse the stability of the proposed BPI controller, $\beta(t)$ is considered as a slowly time-varying parameter with $\beta(t) \in[0, \pi / 2)$ rad. Therefore, in this section, the stability of the azimuth feedback loop with the proposed BPI controller is analysed to determine the maximum value of $\bar{\beta}, \bar{\beta}_{\text {max }}$ such 
that the azimuth feedback loop with the proposed BPI controller is stable for all $\beta(t) \in\left[0, \bar{\beta}_{\max }\right] \operatorname{rad}$.

For the resulting Linear Matrix Inequality (LMI) problems that are described in this section, a bisection search is used to determine $\bar{\beta}_{\max }$ to an arbitrary accuracy.

\subsection{Quadratic Stability Analysis}

\subsubsection{Quadratic Stability Analysis Based on Generic LPV System}

A sufficient and necessary condition for the quadratic stability of generic LPV systems is given as follows:

Theorem 1. Briat, 2015, p. 51); Amato, 2006, p. 31). The generic LPV system (27) is quadratically stable if and only if there exist a matrix $\boldsymbol{P}=\boldsymbol{P}^{T} \succ 0$ such that the $L M I$

$$
A(\rho)^{T} \mathbf{P}+\mathbf{P} A(\rho) \prec 0
$$

holds for all $\rho \in \Delta_{\rho}$.

Theorem 1 does not take into consideration the rate of variation of the parameters, hence, arbitrarily fast variations of the parameters are allowed. This clearly leads to conservative stability conditions when considering systems with slowly time-varying parameters.

Theorem 1 is used to analyse the quadratic stability of the azimuth feedback loop with the proposed BPI controller given in $(28)$, and the matrix, $\mathbf{P}_{\mathbf{g}}$ is defined as:

$$
\mathbf{P}_{\mathbf{g}}=\left[\begin{array}{cc}
P_{g 1} & P_{g 2} \\
P_{g 2} & P_{g 3}
\end{array}\right] \succ 0
$$

Then, the LMI 35 becomes $A(\beta(t))^{T} \mathbf{P}_{\mathbf{g}}+\mathbf{P}_{\mathbf{g}} A(\beta(t)) \prec 0$ and it is further rewritten as:

$$
\left[\begin{array}{cc}
2 P_{g 2} a_{1} f(\beta(t)) & P_{g 1}-P_{g 2} a_{2} f(\beta(t))+P_{g 3} a_{1} f(\beta(t)) \\
\star & -2 P_{g 2}-2 P_{g 3} a_{2} f(\beta(t))
\end{array}\right] \prec 0
$$

LMI (37) is an LMI feasibility problem. With $a=V_{\text {rop }} K_{\mathrm{dls}}=4.6533 \times 10^{-3}$ $\mathrm{rad} / \mathrm{min}, k_{\mathrm{ia}}=0.01, k_{\mathrm{pa}}=0.13$ and $\beta(t) \in\left[0, \bar{\beta}_{\max }\right] \mathrm{rad}$ (with 0.01 increment 
from $0 \mathrm{rad}$ to $\bar{\beta}_{\max } \mathrm{rad}$ ), and using a bisection search and the MATLAB LMI

Toolbox, $\bar{\beta}_{\max }=1.14 \mathrm{rad}$ is obtained and an admissible solution of the matrix, $\mathbf{P}_{\mathbf{g}}$ is obtained as:

$$
\mathbf{P}_{\mathrm{g}}=\left[\begin{array}{cc}
1.8681 \times 10^{-6} & -1.2247 \times 10^{-5} \\
-1.2247 \times 10^{-5} & 3.6967 \times 10^{-2}
\end{array}\right]
$$

Based on (38), LMIs (36) and (37) satisfy Theorem 1 for all $\beta(t) \in[0,1.14]$ rad. Therefore, the azimuth feedback loop with the proposed BPI controller given in $[28)$ is quadratically stable for all $\beta(t) \in[0,1.14] \mathrm{rad}$.

\subsubsection{Quadratic Stability Analysis Based on Polytopic LPV System}

A sufficient and necessary condition for the quadratic stability of polytopic LPV systems is given as follows:

Theorem 2. Briat, 2015, p. 56). The polytopic LPV system 29 is quadratically stable if and only if there exist a matrix $\boldsymbol{P}=\boldsymbol{P}^{T} \succ 0$ such that the LMIs

$$
A_{i}^{T} \mathbf{P}+\mathbf{P} A_{i} \prec 0
$$

hold for all $i=1, \ldots, M$.

Similar to Theorem 1. Theorem 2 does not take into consideration the rate of variation of the parameters, hence, arbitrarily fast variations of the parameters are allowed. This clearly leads to conservative stability conditions when considering systems with slowly time-varying parameters.

Theorem 2 is used to analyse the quadratic stability of the azimuth feedback loop with the proposed BPI controller given in (30) and (31), and the matrix, $\mathbf{P}_{\mathbf{p}}$ is defined as:

$$
\mathbf{P}_{\mathbf{p}}=\left[\begin{array}{cc}
P_{p 1} & P_{p 2} \\
P_{p 2} & P_{p 3}
\end{array}\right] \succ 0
$$

Then, LMIs 39 become: $A_{1}^{T} \mathbf{P}_{\mathbf{p}}+\mathbf{P}_{\mathbf{p}} A_{1} \prec 0$ and $A_{2}^{T} \mathbf{P}_{\mathbf{p}}+\mathbf{P}_{\mathbf{p}} A_{2} \prec 0$, and they are further rewritten as:

$$
\left[\begin{array}{cc}
-2 P_{p 2} a_{1} \underline{p} & P_{p 1}-P_{p 2} a_{2} \underline{p}+P_{p 3} a_{1} \underline{p} \\
\star & -2 P_{p 2}-2 P_{p 3} a_{2} \underline{p}
\end{array}\right] \prec 0
$$




$$
\left[\begin{array}{cc}
-2 P_{p 2} a_{1} \bar{p} & P_{p 1}-P_{p 2} a_{2} \bar{p}+P_{p 3} a_{1} \bar{p} \\
\star & -2 P_{p 2}-2 P_{p 3} a_{2} \bar{p}
\end{array}\right] \prec 0
$$

LMIs 41] and 42 are LMI feasibility problems. With $a=V_{\text {rop }} K_{\mathrm{dls}}=4.6533 \times$ $10^{-3} \mathrm{rad} / \mathrm{min}, k_{\mathrm{ia}}=0.01, k_{\mathrm{pa}}=0.13, \beta(t) \in\left[0, \bar{\beta}_{\text {max }}\right] \mathrm{rad}$ and $p(t) \in[1, \bar{p}]$, and using a bisection search and the MATLAB LMI Toolbox, $\bar{\beta}_{\max }=1.1464 \mathrm{rad}$ and $\bar{p}=1.2041$ are obtained and an admissible solution of the matrix, $\mathbf{P}_{\mathbf{p}}$ is obtained as:

$$
\mathbf{P}_{\mathbf{p}}=\left[\begin{array}{cc}
17.1594 & -1.11506 \times 10^{2} \\
-1.11506 \times 10^{2} & 3.3751 \times 10^{5}
\end{array}\right]
$$

Based on (43), LMIs (40), 41) and 42) satisfy Theorem 2 for all $\beta(t) \in$ $[0,1.1464]$ rad. Therefore, the azimuth feedback loop with the proposed BPI controller given in (30) and (31) is quadratically stable for all $\beta(t) \in[0,1.1464]$ rad.

\subsubsection{Quadratic Stability Analysis Based on Affine Parameter-Dependent Sys-} tem

The quadratic stability of the affine parameter-dependent system, 32 can be analysed by the MATLAB function quadstab which takes into consideration solution of various LMI while attempting to find a positive definite quadratic Lyapunov function of the form:

$$
V(x)=x^{T} \mathbf{P} x
$$

such that $d V(x) / d t<0$ along all state trajectories (Gu et al., 2013, p. 229). For the quadratic Lyapunov function given in 44, the stability condition $d V(x) / d t<0$ is equivalent to

$\left(A_{f 0}+\rho_{1}(t) A_{f 1}+\cdots+\rho_{n}(t) A_{f n}\right)^{T} \mathbf{P}+\mathbf{P}\left(A_{f 0}+\rho_{1}(t) A_{f 1}+\cdots+\rho_{n}(t) A_{f n}\right) \prec 0$

Nonetheless, owing to the fact that arbitrarily fast variations of parameters are allowed by the MATLAB function quadstab, the MATLAB function quadstab 
provides conservative stability results when considering systems with slowly time-varying parameters.

The MATLAB function quadstab is used to analyse the quadratic stability of the azimuth feedback loop with the proposed BPI controller given in (33), and the matrix $\mathbf{P}_{\mathbf{a}}$ is defined as:

$$
\mathbf{P}_{\mathbf{a}}=\left[\begin{array}{cc}
P_{a 1} & P_{a 2} \\
P_{a 2} & P_{a 3}
\end{array}\right] \succ 0
$$

Then, the LMI 45 becomes:

$$
\left(A_{f 0}+p(t) A_{f 1}\right)^{T} \mathbf{P}_{\mathbf{a}}+\mathbf{P}_{\mathbf{a}}\left(A_{f 0}+p(t) A_{f 1}\right) \prec 0
$$

and it is further rewritten as:

$$
\left[\begin{array}{cc}
-2 P_{a 2} a_{1} p(t) & \left.P_{a 1}-P_{a 2} a_{2} p(t)+P_{a 3} a_{1} p(t)\right) \\
\star & -2 P_{a 2}-2 P_{a 3} a_{2} p(t)
\end{array}\right] \prec 0
$$

LMI (48) is an LMI feasibility problem. With $a=V_{\text {rop }} K_{\mathrm{dls}}=4.6533 \times 10^{-3}$ $\mathrm{rad} / \mathrm{min}, k_{\mathrm{ia}}=0.01, k_{\mathrm{pa}}=0.13$ and $\beta(t) \in\left[0, \bar{\beta}_{\max }\right] \mathrm{rad}$, and using a bisection search and the MATLAB function quadstab, $\bar{\beta}_{\max }=1.1464 \mathrm{rad}$ is obtained and an admissible solution of the matrix, $\mathbf{P}_{\mathbf{a}}$ is obtained as:

$$
\mathbf{P}_{\mathbf{a}}=\left[\begin{array}{cc}
3.9125 \times 10^{-6} & -2.5457 \times 10^{-5} \\
-2.5457 \times 10^{-5} & 7.6956 \times 10^{-2}
\end{array}\right]
$$

Based on (49), LMIs (46) and (48) satisfy LMI 45) for all $\beta(t) \in[0,1.1464]$ rad. Therefore, the azimuth feedback loop with the proposed BPI controller given in (33) is quadratically stable for all $\beta(t) \in[0,1.1464]$ rad.

Comparing the quadratic stability results obtained from Theorems 1 and 2 and the MATLAB function quadstab, $\bar{\beta}_{\max }=1.14 \mathrm{rad}$ is obtained from Theorem 1 and $\bar{\beta}_{\max }=1.1464 \mathrm{rad}$ is obtained from Theorem 2 and the MATLAB function quadstab (also see Table 2). Hence, a more conservative stability result is obtained from Theorem 1 than that obtained from Theorem 2 and the MATLAB function quadstab.

The stability notions for the generic LPV, polytopic LPV and affine parameterdependent systems to be quadratically stable considered in this subsection does 
not take into consideration the rate of variation of the parameters, hence, arbitrarily fast variations of the parameters are allowed. As expected, this clearly leads to conservative stability conditions while considering systems with slowly time-varying parameters, such as 25. To reduce the conservatism of the quadratic stability, the subsequent subsection presents the analysis of robust stability which takes into consideration the rate of variation of the parameters.

\subsection{Robust Stability Analysis}

\subsubsection{Robust Stability Analysis Based on Affine Parameter-Dependent System}

The robust stability of the affine parameter-dependent system, 32 can be analysed by the MATLAB function pdlstab which takes into consideration solution of various LMIs while attempting to find a positive definite parameterdependent Lyapunov function of the form:

$$
V(x, \rho)=x^{T} Q(\rho)^{-1} x
$$

where $Q(\rho)=Q_{0}+\rho_{1} Q_{1}+\rho_{2} Q_{2}+\cdots+\rho_{n} Q_{n}$ and $Q_{0}, Q_{1}, Q_{2}, \ldots, Q_{n}$ are symmetric matrices, such that $d V(x, \rho) / d t<0$ along all admissible trajectories (Gu et al., 2013, p. 229). For the parameter-dependent Lyapunov function given in 50, the stability condition $d V(x, \rho) / d t<0$ is equivalent to

$$
\begin{aligned}
& Q(\rho)\left(A_{f 0}+\rho_{1}(t) A_{f 1}+\cdots+\rho_{n}(t) A_{f n}\right)^{T} \\
& \quad+\left(A_{f 0}+\rho_{1}(t) A_{f 1}+\cdots+\rho_{n}(t) A_{f n}\right) Q(\rho)-\frac{d Q(\rho)}{d t} \prec 0
\end{aligned}
$$

In the case of systems with slowly varying or constant parameters, less conser-

vative stability results could be obtained by the MATLAB function pdlstab (Gu et al. 2013, p. 229).

The MATLAB function pdlstab is used to analyse the robust stability of the azimuth feedback loop with the proposed BPI controller given in (33), and the matrix $Q(p(t))$ is defined as:

$$
Q(p(t))=Q_{0}+p(t) Q_{1} \succ 0
$$


Then, the LMI 51 becomes:

$$
Q(p(t))\left(A_{f 0}+p(t) A_{f 1}\right)^{T}+\left(A_{f 0}+p(t) A_{f 1}\right) Q(p(t))-\frac{d Q(p(t))}{d t} \prec 0
$$

LMI (53) is an LMI feasibility problem. With $a=V_{\text {rop }} K_{\mathrm{dls}}=4.6533 \times 10^{-3}$ $\mathrm{rad} / \mathrm{min}, k_{\mathrm{ia}}=0.01, k_{\mathrm{pa}}=0.13$ and $\beta(t) \in\left[0, \bar{\beta}_{\max }\right] \mathrm{rad}$, and using a bisection search and the MATLAB function pdlstab, $\bar{\beta}_{\max }=1.1464 \mathrm{rad}$ is obtained and an admissible solution of the matrices, $Q_{0}$ and $Q_{1}$ are obtained as:

$$
Q_{0}=\left[\begin{array}{cc}
9.5986 \times 10^{6} & 3.1746 \times 10^{3} \\
3.1746 \times 10^{3} & 4.88 \times 10^{2}
\end{array}\right], \quad Q_{1}=\left[\begin{array}{cc}
-7.1775 & 0.0929 \\
0.0929 & 0.0019
\end{array}\right]
$$

Based on (54), LMIs (52) and (53) are satisfied for all $\beta(t) \in[0,1.1464]$. Therefore, the azimuth feedback loop with the proposed BPI controller given in (33) is robustly stable for all $\beta(t) \in[0,1.1464]$ rad.

\subsubsection{Robust Stability Analysis Based on Polytopic LPV System}

The rate of variation of the parameters is captured in the term $\dot{\lambda}$. To define a set that contains the trajectories of $\dot{\lambda}$, it is assumed that the polytopic LPV system, 29) approximates the generic LPV system, 27) with $M_{p}$ parameters, given by $\rho$, where the bounds of the derivative are known (Briat, 2015. p. 57). Hence, a polytope where $\dot{\lambda}$ evolves within is defined from the following proposition:

Proposition 1. Briat, 2015, p. 58). Assume that $\dot{\rho} \in \Delta_{\nu}=\boldsymbol{c o}\left\{\boldsymbol{V}_{\nu}\right\}, \boldsymbol{V}_{\nu}=$ $\left\{d_{1}, \ldots, d_{M}\right\}, M=2^{M_{p}}$ and that the decomposition

$$
\rho(t)=\sum_{i=1}^{M} \lambda_{i}(t) v_{i}
$$

holds with $\boldsymbol{V}_{\rho}=\left\{v_{1}, \ldots, v_{M}\right\}$. Then, the set of all $\dot{\lambda}$ 's is given by

$$
\dot{\Lambda}_{M}:=\left\{\left[\begin{array}{c}
\mathbf{V} \\
\mathbf{1}_{M}^{T} \\
\mathbf{0}_{M}
\end{array}\right]^{+}\left[\begin{array}{c}
\mathbf{D} \\
\mathbf{0}_{M} \\
\mathbf{1}_{M}^{T}
\end{array}\right] \zeta: \zeta \in \Lambda_{M}\right\}
$$


where $\mathbf{1}_{M}$ denotes a column vector of dimension $M$ containing 1 entries, $\mathbf{0}_{M}$ denotes a row vector of dimension $M$ containing 0 entries, $\mathbf{D}$ denotes a set of vertices, $d_{i}$, that is, $\mathbf{D}=\left[d_{1}, \ldots, d_{M}\right], \mathbf{V}=\left[v_{1}, \ldots, v_{M}\right]$ and $\zeta(t) \in \Lambda_{M}$. In addition, the following identity is obtained:

$$
\dot{\rho}(t)=\sum_{i=1}^{M} \dot{\lambda}_{i}(t) v_{i}=\sum_{i=1}^{M} \zeta_{i}(t) d_{i}
$$

From Proposition 1 a matrix of vertex points, $\operatorname{vert}\left\{\dot{\Lambda}_{M}\right\}$ can be defined as:

$$
\mathbf{L}=\left[\begin{array}{c}
\mathbf{V} \\
\mathbf{1}_{M}^{T} \\
\mathbf{0}_{M}
\end{array}\right]^{+}\left[\begin{array}{c}
\mathbf{D} \\
\mathbf{0}_{M} \\
\mathbf{1}_{M}^{T}
\end{array}\right]
$$

The robust stability of the polytopic system, 290 can be analysed by the following theorem:

Theorem 3. Briat, 2015, p. 61). The polytopic LPV system 29 is robustly stable if there exist matrices $\boldsymbol{P}_{i}=\boldsymbol{P}_{i}^{T} \succ 0, i=1, \ldots, M$, a matrix $\boldsymbol{X} \in \mathbb{R}^{n \times n}$ and a sufficiently large scalar $\xi>0$ such that the matrix inequalities

$$
\left[\begin{array}{ccc}
-\left(\mathbf{X}+\mathbf{X}^{T}\right) & \mathbf{P}_{i}+\mathbf{X}^{T} A_{i} & \mathbf{X}^{T} \\
\star & -\xi \mathbf{P}_{i}+\sum_{j=1}^{M} \mathbf{P}_{j} l_{j i} & 0 \\
\star & \star & -\mathbf{P}_{i} / \xi
\end{array}\right] \prec 0
$$

hold for all $i=1, \ldots, M$, where $l_{j i}$ are the elements of $\mathbf{L}$.

Theorem 3 takes into consideration the rate of variation of the parameters, hence, provides less conservative stability results.

Theorem 3 is used to analyse the robust stability of the polytopic LPV system given in (30) and (31). Based on Theorem 3 , the rate of variation of the parameters is captured in the term $\dot{\lambda}$. To define a set that contains the trajectories of $\dot{\lambda}$, it is assumed that the polytopic LPV system, 30 and 31 approximates the generic LPV system, 28 with $M_{p}$ parameters, given by $\rho$, where the bounds of the derivative are known. Hence, a polytope where $\dot{\lambda}$ evolves within is defined from Proposition 1. 
In this case, and with reference to Proposition 1 and $(58), \mathbf{V}=p(t)=[\underline{p}, \bar{p}]$, $\mathbf{D}=\dot{p}(t)=[\underline{\dot{p}}, \overline{\dot{p}}], \overline{\dot{p}}=(\overline{d p} / d \beta) \overline{\dot{\beta}}$ and $\underline{\dot{p}}=-(\overline{d p} / d \beta) \overline{\dot{\beta}}$; where $\overline{d p} / d \beta$ is the maximum value of $d p / d \beta$ and $\overline{\dot{\beta}}$ is the maximum value of $\dot{\beta}$ and it is considered as the maximum possible build rate, that is, $\overline{\dot{\beta}}=V_{\text {rop }} K_{\text {dls }}$. Furthermore, for a time-varying single parameter system such as (30) and (31), $M_{p}=1$ and $M=2^{M_{p}}=2$, the resulting form of $(58)$ evaluation is:

$$
\mathbf{L}=\left[\begin{array}{ll}
l_{11} & l_{12} \\
l_{21} & l_{22}
\end{array}\right]
$$

where $l_{11}, l_{12}, l_{21}$ and $l_{22}$ are the elements of $\mathbf{L}$.

Based on Theorem 3 and the azimuth feedback loop with the proposed BPI controller given in (30) and (31), and given the matrices, $\mathbf{P}_{\mathbf{1}}, \mathbf{P}_{\mathbf{2}}$ and $\mathbf{X}$, the LMIs (59) become:

$$
\begin{gathered}
{\left[\begin{array}{ccc}
-\left(\mathbf{X}+\mathbf{X}^{T}\right) & \mathbf{P}_{\mathbf{1}}+\mathbf{X}^{T} A_{1} & \mathbf{X}^{T} \\
\star & -\xi \mathbf{P}_{\mathbf{1}}+\mathbf{P}_{\mathbf{1}} l_{11}+\mathbf{P}_{\mathbf{2}} l_{21} & 0 \\
\star & \star & -\mathbf{P}_{\mathbf{1}} / \xi
\end{array}\right] \prec 0} \\
{\left[\begin{array}{ccc}
-\left(\mathbf{X}+\mathbf{X}^{T}\right) & \mathbf{P}_{\mathbf{2}}+\mathbf{X}^{T} A_{2} & \mathbf{X}^{T} \\
\star & -\xi \mathbf{P}_{\mathbf{2}}+\mathbf{P}_{\mathbf{1}} l_{12}+\mathbf{P}_{\mathbf{2}} l_{22} & 0 \\
\star & \star & -\mathbf{P}_{\mathbf{2}} / \xi
\end{array}\right] \prec 0}
\end{gathered}
$$

LMIs 61 - 62 are LMI feasibility problems. With $a=\overline{\dot{\beta}}=V_{\mathrm{rop}} K_{\mathrm{dls}}=$ $4.6533 \times 10^{-3} \mathrm{rad} / \mathrm{min}, k_{\mathrm{ia}}=0.01, k_{\mathrm{pa}}=0.13, \beta(t) \in\left[0, \bar{\beta}_{\text {max }}\right] \mathrm{rad}$ and $p(t) \in$ $[1, \bar{p}]$, and using a line-search algorithm 1 , a bisection search and the MATLAB LMI Toolbox, the following are obtained: $\xi=0.9, \bar{\beta}_{\max }=1.5707 \mathrm{rad}, \bar{p}=$ $2964.8, \underline{\dot{p}}=-143200, \overline{\dot{p}}=143200, l_{11}=l_{22}=48.3171$ and $l_{12}=l_{21}=-48.3171$.

\footnotetext{
${ }^{1} \mathrm{~A}$ line-search algorithm continuously increases the value of $\xi$ until the problem becomes feasible, or terminates when the value of $\xi$ exceeds a particular limit value.
} 
Also, admissible solutions of the matrices, $\mathbf{P}_{\mathbf{1}}, \mathbf{P}_{\mathbf{2}}$ and $\mathbf{X}$ are obtained as follows:

$$
\begin{aligned}
\mathbf{P}_{\mathbf{1}} & =\left[\begin{array}{cc}
86.2156 & -60.6632 \\
-60.6632 & 317.9908
\end{array}\right], \quad \mathbf{P}_{\mathbf{2}}=\left[\begin{array}{cc}
86.4084 & -62.1581 \\
-62.1581 & 322.5416
\end{array}\right], \\
\mathbf{X} & =\left[\begin{array}{cc}
89.7116 & 11.7048 \\
-92.0640 & 175.3409
\end{array}\right]
\end{aligned}
$$

320

The matrices, $\mathbf{P}_{\mathbf{1}}, \mathbf{P}_{\mathbf{2}}$ and $\mathbf{X}$ given in 63 and 64 , and $\xi=0.9$ satisfy Theorem 3 for all $\beta(t) \in[0,1.5707] \mathrm{rad}$. Therefore, the azimuth feedback loop with the proposed BPI controller given in (30) and (31) is robustly stable for all $\beta(t) \in[0,1.5707] \mathrm{rad}$.

Comparing the robust stability results obtained from the MATLAB function pdlstab and Theorem $3, \bar{\beta}_{\max }=1.1464 \mathrm{rad}$ is obtained from the MAT$\mathrm{LAB}$ function pdlstab and $\bar{\beta}_{\max }=1.5707 \mathrm{rad}$ is obtained from Theorem 3 . Unexpectedly, for this particular case, the MATLAB function pdlstab gives a conservative stability result as against an expected less conservative stability result and it is the same result as those obtained from Theorem 2 and the MAT-

330 LAB function quadstab (in Subsection 4.1) as shown in Table 2. Expectedly, Theorem 3 gives a less conservative stability result as the stability result proves that the azimuth feedback loop with the proposed BPI controller is robustly stable for nearly the whole range of operations, $\beta(t) \in[0, \pi / 2) \mathrm{rad}$, within practical engineering accuracy.

\begin{tabular}{ll} 
Table $2: \bar{\beta}_{\max }$ for Different LPV Stability Notions \\
LPV Stability Notion & $\bar{\beta}_{\text {max }}$ \\
\hline Theorem 2.2 & $1.1400 \mathrm{rad}$ \\
Theorem 2.3 & $1.1464 \mathrm{rad}$ \\
MATLAB function quadstab & $1.1464 \mathrm{rad}$ \\
MATLAB function pdlstab & $1.1464 \mathrm{rad}$ \\
Theorem 2.4 & $1.5707 \mathrm{rad}$ \\
\hline
\end{tabular}

Based on the fact that the inclination feedback loop with the PI controller is shown to be exponentially stable and the azimuth feedback loop with the pro- 
posed BPI controller is proven to be quadratically and robustly stable, therefore, the proposed BPI control system is proven to be stable.

\section{Simulation Results}

In this section, the transient simulations are carried out using MATLAB/Simulink. The system delays are implemented as $e^{-\tau_{d} s}$, where $\tau_{d}$ is the time delay. The $\tau_{d}$ is dependent on the $V_{\text {rop }}$ and the distance of the D\&I sensor from the bit, $d_{t}$ which is given by

$$
\tau_{d}=\frac{d_{t}}{V_{\text {rop }}}
$$
mented to investigate the invariance of the azimuth responses of the proposed BPI controller and the PI controller proposed by Panchal et al. (2010).

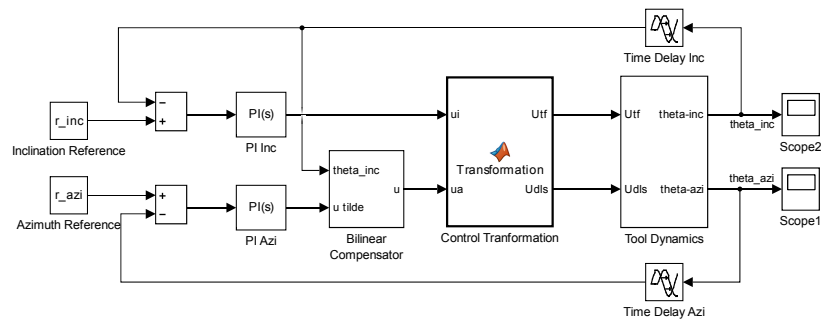

Figure 9: Simulink diagram of LFM simulation scheme for BPI controller

Based on the LFM simulation, the PI controller proposed by Panchal et al. (2010) and the proposed BPI controller azimuth responses for a reference change 
Table 3: Simulation Parameters for BPI Controller with LFM

\begin{tabular}{|l|c||l|c|}
\hline Parameter & Value & Parameter & Value \\
\hline$\theta_{\text {inc }}$ & $\pi / 6 \mathrm{rad}\left(30^{\circ}\right)$ & $k_{\mathrm{ii}}$ & 0.01 \\
\hline$\theta_{\mathrm{azi}}$ & $200 \mathrm{ft} / \mathrm{hr}(1.0158 \mathrm{~m} / \mathrm{min})$ & $k_{\mathrm{pa}}$ & 0.13 \\
\hline$V_{\mathrm{rop}}$ & $8^{\circ} / 100 \mathrm{ft}(4.5809 \mathrm{e}-3 \mathrm{rad} / \mathrm{m})$ & $k_{\mathrm{pi}}$ & 0.01 \\
\hline$K_{\mathrm{dls}}$ & $1^{\circ} / 100 \mathrm{ft}(5.7261 \mathrm{e}-4 \mathrm{rad} / \mathrm{m})$ & $r_{\mathrm{inc}}$ & $\pi / 6+0.015 \mathrm{rad}$ \\
\hline$V_{\mathrm{dr}}$ & $0.5^{\circ} / 100 \mathrm{ft}(2.8631 \mathrm{e}-4 \mathrm{rad} / \mathrm{m})$ & $r_{\mathrm{azi}}$ & $\pi / 6+0.015 \mathrm{rad}$ \\
\hline$V_{\mathrm{tr}}$ & $14.997 \mathrm{ft}(4.5711 \mathrm{~m})$ & $\tau_{d}$ & $4.5 \mathrm{~min}$ \\
\hline$d_{t}$ & & \\
\hline
\end{tabular}

of $0.015 \mathrm{rad}$ for a set of various azimuth angles are shown in Figs. 10 and 11 respectively, where $\Delta \theta_{\text {azi }}(t)=\theta_{\text {azi }}(t)-\theta_{\text {azi }}(0)$ and $\tau_{d}=0$ min. Comparing the azimuth responses of the PI controller proposed by Panchal et al. (2010) and the proposed BPI controller, the proposed BPI controller azimuth responses tend to converge more closely to the nominal operating point of $\pi / 2 \mathrm{rad}$, than the PI controller proposed by Panchal et al. (2010) azimuth responses. Hence, the proposed BPI controller provides more consistent azimuth responses over wider range of the directional drilling tool operations, than the PI controller proposed by Panchal et al. (2010).

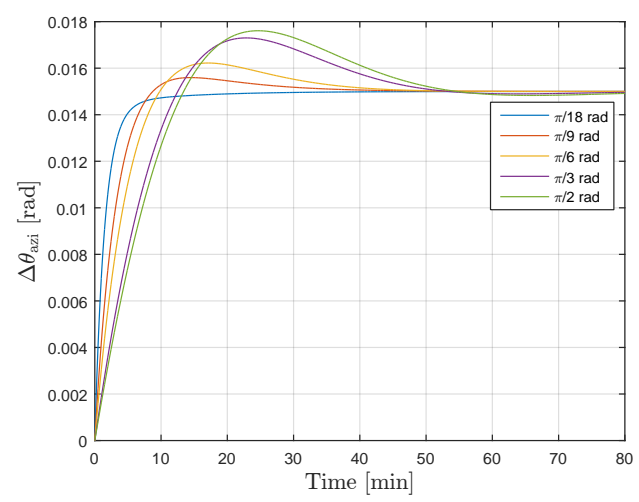

Figure 10: PI controller (proposed by Panchal et al. (2010)) azimuth response with LFM 


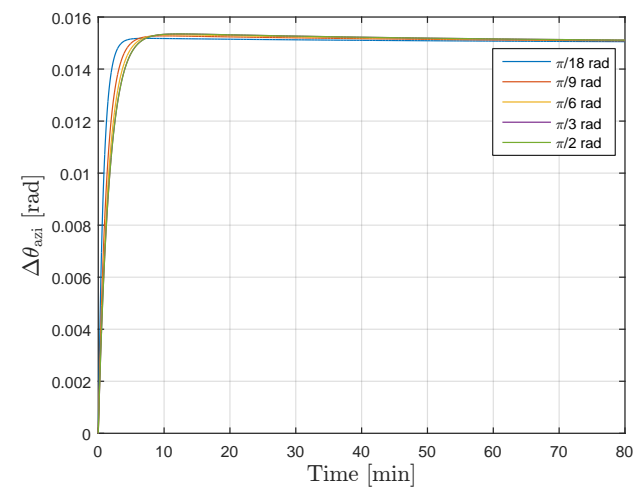

Figure 11: BPI controller azimuth response with LFM
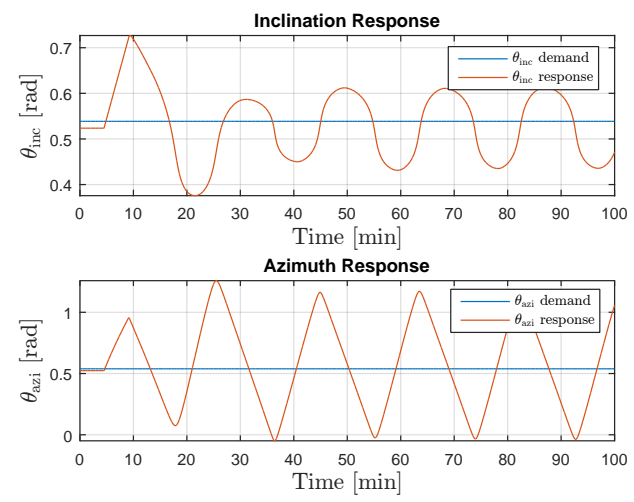

Figure 12: PI controller (proposed by Panchal et al. (2010)) attitude response with LFM

To investigate the adverse effects of disturbances and time delay on the feedback measurements with respect to the stability and performance of the directional drilling tool, the inclination and azimuth responses to step changes of the PI controller proposed by Panchal et al. (2010) and the proposed BPI controller based on the LFM simulation are shown in Figs. 12 and 13 , respectively.

The inclination and azimuth responses to step changes, from $\pi / 6 \mathrm{rad}$ to $\pi / 6+0.015 \mathrm{rad}$ and from $\pi / 6$ to $\pi / 6+0.015 \mathrm{rad}$, respectively, of the PI controller proposed by Panchal et al. (2010) are shown in Fig. 12. The attitude response of the PI controller proposed by Panchal et al. (2010) exhibits oscillatory characteristics. Hence, the inclination and azimuth responses of the 

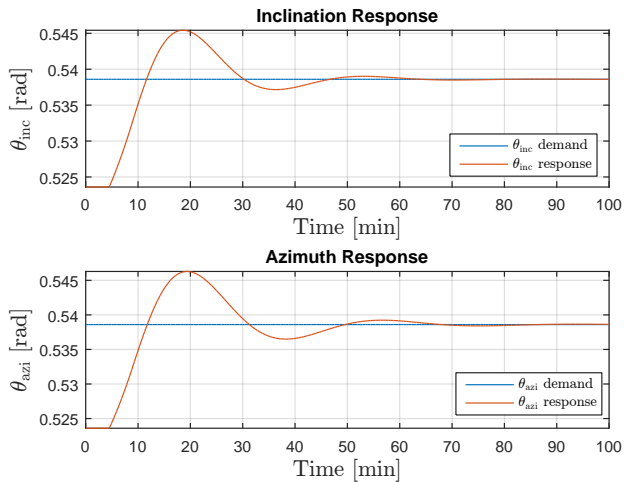

Figure 13: BPI controller attitude response with LFM
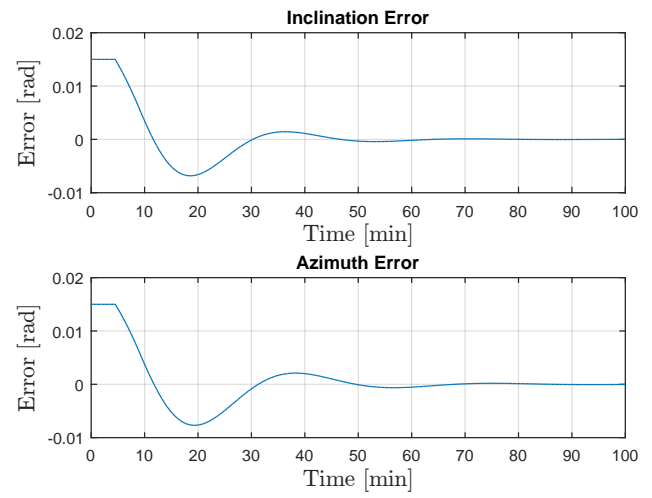

Figure 14: Attitude error for BPI controller with LFM

directional drilling tool do not converge to the desired angles of $\pi / 6+0.015 \mathrm{rad}$ and $\pi / 6+0.015 \mathrm{rad}$, respectively, as the PI controller proposed by Panchal et al. (2010) is unable to handle the adverse effects of the drop rate disturbance of up to $1^{\circ} / 100 \mathrm{ft}$, turn rate bias disturbance of up to $0.5^{\circ} / 100 \mathrm{ft}$ and time delay of up to $4.5 \mathrm{~min}$ on the feedback measurements.

The inclination and azimuth responses to step changes, from $\pi / 6 \mathrm{rad}$ to $\pi / 6+0.015 \mathrm{rad}$ and from $\pi / 6 \mathrm{rad}$ to $\pi / 6+0.015 \mathrm{rad}$, respectively, of the proposed BPI controller are shown in Fig. 13. The inclination and azimuth responses of the directional drilling tool converges to the desired angles of $\pi / 6+0.015 \mathrm{rad}$ and $\pi / 6+0.015 \mathrm{rad}$, respectively. Hence, the proposed BPI controller reduces 
the adverse effects of the drop rate disturbance of up to $1^{\circ} / 100 \mathrm{ft}$, turn rate bias disturbance of up to $0.5^{\circ} / 100 \mathrm{ft}$ and time delay of up to $4.5 \mathrm{~min}$ on the feedback measurements with respect to the stability and performance, than the PI controller proposed by Panchal et al. (2010).

The inclination and azimuth errors for the proposed BPI controller are shown in Fig. 14. The proposed BPI controller is able to converge the inclination and azimuth errors directly to zero within $62 \mathrm{~min}$.

\subsection{High-Fidelity Model Simulation}

Using the nonlinear system given by (2) and (3), the proposed BPI controller, the systems delays and the drilling cycle scheme presented in Inyang (2017), MATLAB/Simulink transient simulations are created based on the HFM simulation scheme, shown in Fig. 15, to further analyse the effectiveness, robustness and stability of the proposed BPI controller. The parameters used for the HFM simulations are given in Table 4 . The values of $\tau_{d}, k_{\mathrm{ia}}, k_{\mathrm{pa}}, k_{\mathrm{ii}}, k_{\mathrm{pi}}, V_{\mathrm{dr}}, V_{\mathrm{tr}}$ and $d_{t}$ are the same as those for the LFM simulation (see Table 3).

Table 4: Simulation Parameters for BPI Controller with HFM

\begin{tabular}{|l|c|l|l|}
\hline Parameter & Value & Parameter & Value \\
\hline$V_{\text {rop }}$ & $200 \mathrm{ft} / \mathrm{hr}(1.0158 \mathrm{~m} / \mathrm{min})$ & $\theta_{\text {inc }}$ & $\pi / 2 \mathrm{rad}\left(90^{\circ}\right)$ \\
\hline$K_{\mathrm{dls}}$ & $8^{\circ} / 100 \mathrm{ft}(4.5809 \mathrm{e}-3 \mathrm{rad} / \mathrm{m})$ & $\theta_{\text {azi }}$ & $\pi / 2 \mathrm{rad}\left(90^{\circ}\right)$ \\
\hline$T_{\mathrm{a}}$ & $0.05 \mathrm{~s}(8.333 \mathrm{e}-4 \mathrm{~min})$ & $r_{\mathrm{inc}}$ & $\pi / 2+0.015 \mathrm{rad}$ \\
\hline$k_{\mathrm{vi}}$ & $6 \mathrm{~s}^{-1}\left(360 \mathrm{~min}^{-1}\right)$ & $r_{\mathrm{azi}}$ & $\pi / 2+0.015 \mathrm{rad}$ \\
\hline$\hat{\omega}_{\mathrm{tf}}$ & $2 \pi \mathrm{rad} / \mathrm{s}(376.991 \mathrm{rad} / \mathrm{min})$ & $k_{\mathrm{p}}$ & $1 \mathrm{~s}^{-1}\left(60 \mathrm{~min}^{-1}\right)$ \\
\hline$t_{\text {cycle }}$ & $10 \mathrm{~s}(0.1667 \mathrm{~min})$ & $k_{\mathrm{vp}}$ & 0.5 \\
\hline
\end{tabular}

Based on the HFM simulation, the inclination and azimuth responses to step changes, from $\pi / 2 \mathrm{rad}$ to $\pi / 2+0.015 \mathrm{rad}$ and from $\pi / 2 \mathrm{rad}$ to $\pi / 2+0.015 \mathrm{rad}$, respectively, of the proposed BPI controller are shown in Fig. 16 . The proposed

BPI controller still holds the inclination and azimuth of the directional drilling 


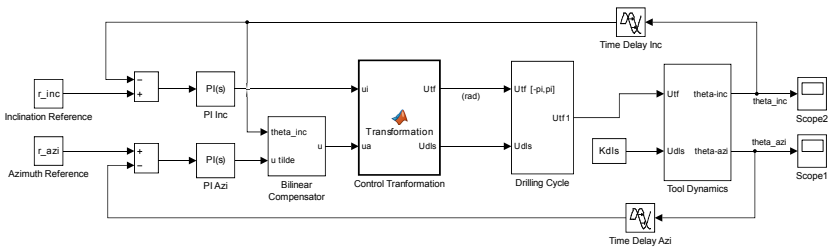

Figure 15: Simulink diagram of HFM simulation scheme for BPI controller
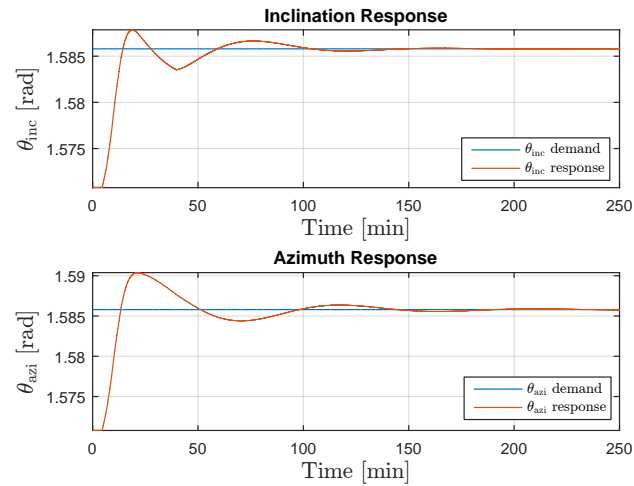

Figure 16: BPI controller attitude response with HFM
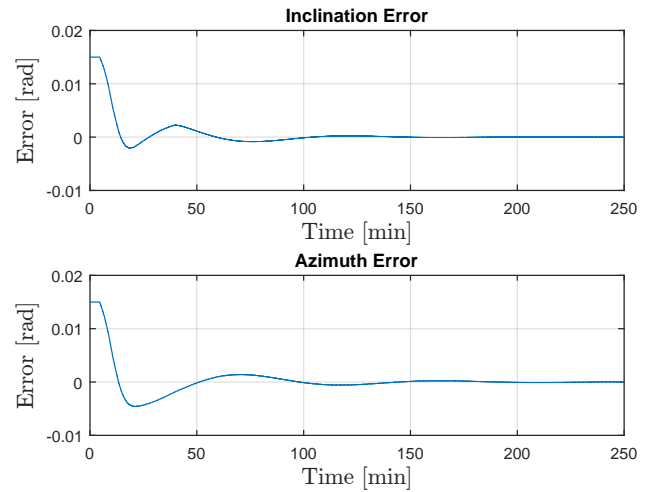

Figure 17: Attitude error for BPI controller with HFM

tool at the desired angles of $\pi / 2+0.015 \mathrm{rad}$ and $\pi / 2+0.015 \mathrm{rad}$, respectively. Hence, the proposed BPI controller is effective, stable and robust to handle the adverse effects of the drop rate disturbance of up to $1^{\circ} / 100 \mathrm{ft}$, turn rate bias disturbance of up to $0.5^{\circ} / 100 \mathrm{ft}$, time delay of up to $4.5 \mathrm{~min}$ on the feedback measurements and some other uncertainties (such as drilling cycle and toolface 
actuator dynamics) in the attitude control of the directional drilling tool.

The inclination and azimuth errors for the proposed BPI controller with the HFM are shown in Fig. 17. The proposed BPI controller is able to converge the inclination and azimuth errors directly to zero within $150 \mathrm{~min}$.

\section{Conclusions}

This paper proposes a bilinear model of directional drilling tool. The proposed bilinear model characterises the nonlinear properties of directional drilling tool more accurately than the existing linear model. Hence, it broadens the range of adequate performance and it is considered to be significantly advanmodel is used as the basis for the design of a Bilinear Proportional plus Integral (BPI) controller for the attitude control of directional drilling tool.

The proposed BPI control system is proven to be stable using stability notions for LTI and LPV systems. The proposed BPI controller is able hold the attitude of the directional drilling tool at the desired inclination and azimuth angles, provide more consistent azimuth responses over wider range of directional drilling operations and significantly reduces the adverse effects of time delay of up to $4.5 \mathrm{~min}$ on the feedback measurements, drop rate disturbance of up to $1^{\circ} / 100 \mathrm{ft}$, turn rate bias disturbance of up to $0.5^{\circ} / 100 \mathrm{ft}$, and some other uncertainties (such as drilling cycle and toolface actuator dynamics) in the attitude control of the directional drilling tool. However, the proposed BPI controller is restricted to attitudes such that $\theta_{\text {inc }}$ is not close to $0^{\circ}$, and also, the performance of the proposed BPI controller reduces as the time delay, drop rate disturbance and turn rate bias disturbance increases above $4.5 \mathrm{~min}, 1^{\circ} / 100$ 430

The proposed BPI controller is relatively simple and it can easily be implemented in the directional drilling tools to increase the potential of accessing difficult reservoirs, drilling more effectively and cost-effective field development. The implementation of the proposed BPI controller should reduce the workload 
of the directional driller because with the significant reduction of the adverse effects of measurement delay and disturbances during directional drilling operations, the directional drilling tool will drill more effectively, hence a less intervention is required from the directional driller.

Stability proof of the proposed BPI controller with time delay remains an 440 open problem.

\section{Acknowledgements}

The authors are grateful to Schlumberger for previous support of the development of the initial attitude controller.

The authors are thankful to Dr Corentin Briat for his advice on the robust stability analysis of the polytopic LPV system.

\section{References}

Al-zahrani, A.A., Wali, M.M., 1994. An adaptive bilinear model-predictive control algorithm for a plate heat exchanger. Developments in Chemical Engineering and Mineral Processing $2,158-170$.

Amato, F., 2006. Robust Control of Linear Systems Subject to Uncertain Time-Varying Parameters. volume 325. Springer, Berlin.

Baker, R., 1996. A Primer of Oilwell Drilling. 5th (revised) ed., The University of Texas at Austin in cooperation with International Association of Drilling Contractors, Texas, USA.

Bayliss, M.T., Inyang, I.J., Whidborne, J.F., 2015. Application of LQG control to attitude 455 control of directional drilling, in: 24th International Conference on Systems Engineering, Coventry, UK.

Bayliss, M.T., Whidborne, J.F., 2015. Mixed uncertainty analysis of pole placement and $\mathrm{H}_{\infty}$ controllers for directional drilling attitude tracking. Journal of Dynamic Systems, Measurement, and Control 137, 121008.

Briat, C., 2015. Linear Parameter-Varying and Time-Delay Systems: Analysis, Observation, Filtering \& Control. Springer, Berlin, Heidelberg.

Bruni, C., DiPillo, G., Koch, G., 1974. Bilinear systems: An appealing class of nearly linear systems in theory and applications. IEEE Transactions on Automatic Control AC-19, $334-348$. 
Burnham, K.J., Dunoyer, A., Marcroft, S., 1999. Bilinear controller with PID structure. Computing and Control Engineering Journal 10, 63-69.

Chen, Y.P., Chang, J.L., Lai, K.M., 2000. Stability analysis and bang-bang sliding control of a class of single-input bilinear systems. IEEE Transactions on Automatic Control 45, $2150-2154$.

Cockburn, C., Matheus, J., Dang, K.L.P., 2011. Automatic trajectory control in extendedreach wells, in: SPE Middle East Oil and Gas Show and Conference, Manama, Bahrain.

Devereux, S., 1999. Drilling Technology in Nontechnical Language. PennWell Corporation, OK.

Downton, G.C., 2012. Challenges of modeling drilling systems for the purposes of automation and control, in: IFAC Workshop on Automatic Control in Offshore Oil and Gas Production, Trondheim, Norway.

Downton, G.C., 2014. Systems modeling and design of automated-directional-drilling systems, in: SPE Annual Technical Conference and Exhibition, Amsterdam, Netherlands.

Gahinet, P., Apkarian, P., Chilali, M., 1994. Affine parameter-dependent lyapunov functions for real parameteric uncertainty, in: 33rd Conference on Decision and Control, FL.

Gahinet, P., Apkarian, P., Chilali, M., 1996. Affine parameter-dependent lyapunov functions and real parameteric uncertainty. IEEE Transactions on Automatic Control 41, 436-442.

Genevois, J., Boulet, J., Simon, C., Reullon, C., 2003. Gyrostab project: The missing link azimuth and inclination mastered with new principles for standard rotary BHAs, in: SPE/IADC Drilling Conference, Amsterdam, Netherlands.

Ghasemi, M., Ibrahim, A., Gildin, E., 2014. Reduced order modeling in reservoir simulation using the bilinear approximation techniques, in: SPE Latin American and Caribbean Petroleum Engineering Conference, Maracaibo, Venezuela.

Goodhart, S.G., Burnham, K.J., James, D.J.G., 1994. Bilinear self-tuning control of a high temperature heat treatment plant. IEEE Proceedings: Control Theory and Applications $141,12-18$.

Gu, D.W., Petkov, P.H., Konstantinov, M.M., 2013. Robust Control Design with MATLAB. 2nd ed., Springer Verlag, London.

Inyang, I.J., 2017. Attitude Control of Directional Drilling. Ph.D. thesis. Cranfield University. 
Inyang, I.J., Whidborne, J.F., Bayliss, M.T., 2016. Bilinear modelling and bilinear PI control of directional drilling, in: 11th UKACC International Conference on Control, Belfast, UK.

Jijón, R.B., Canudas-de-Wit, C., Niculescu, S.I., Dumon, J., 2010. Adaptive observer design under low data rate transmission with applications to oil well drill-string, in: American Control Conference, Baltimore, MD.

Kelman, A., Borrelli, F., 2011. Bilinear model predictive control of a HVAC system using sequential quadratic programming, in: 18th IFAC World Congress, Milan, Italy. pp. 98969874.

Kim, B., Lim, M., 2003. Robust $\mathrm{H}_{\infty}$ control method for bilinear systems. International Journal of Control, Automation and Systems 1, 171-177.

Kremers, N.A.H., Detournay, E., van de Wouw, N., 2016. Model-based robust control of directional drilling systems. IEEE Transactions on Control Systems Technology 24, 226239.

Krener, A.J., 1974. Linearization and bilinearization of control systems, in: Proc. 12th Allerton Conference on Circuits and Systems, Monticello, IL. pp. 834-843.

Kuwana, S., Kiyosawa, Y., Ikeda, A., 1994. Attitude control device and drilling-direction control device. Patent US5307885 A.

Lim, D., Rasmussen, B.P., Swaroop, D., 2009. Selecting PID control gains for nonlinear HVAC\&R systems. HVAC\&R Research 15, 991-1019.

Martineau, S., Burnham, K.J., Haas, O.C.L., Andrews, G., Heeley, A., 2004. Four-term bilinear PID controller applied to an industrial furnace. Control Engineering Practice 12, $457-464$.

Martineau, S., Burnham, K.J., Minihan, J.A., Marcroft, S., Andrews, G., Heeley, A., 2002. Application of a bilinear PID compensator to an industrial furnace, in: 15th IFAC World Congress on Automatic Control, Barcelona, Spain.

Matheus, J., Ignova, M., Hornblower, P., 2012. A hybrid approach to closed-loop directional drilling control using rotary steerable systems, in: Proceedings of the 2012 IFAC Workshop on Automatic Control in Offshore Oil and Gas Production, Trondheim, Norway.

Matheus, J., Ignova, M., Hornblower, P., 2014. A hybrid approach to closed-loop directional drilling control using rotary steerable systems, in: SPE Latin American and Caribbean Petroleum Engineering Conference, Maracaibo, Venezuela.

Matheus, J., Naganathan, S., 2010. Drilling automation: Novel trajectory control algorithms for RSS, in: IADC/SPE Drilling Conference and Exhibition, New Orleans, LA. 
Panchal, N., 2013. Autonomous Trajectory Control for Directioanal Drilling. Ph.D. thesis. Cranfield University. Cranfield, UK.

Panchal, N., Bayliss, M.T., Whidborne, J.F., 2010. Robust linear feedback control of attitude for directional drilling tools, in: 13th IFAC Symposium on Automation in Mining, Mineral and Metal Processing, Cape Town, South Africa.

Park, R.W., Lee, K.T., 2009. Design of a bilinear robust controller for a hydrostatic driver, in: IEEE International Symposium on Industrial Electronics, Seoul, Korea. pp. 1470-1475.

Pedersen, M., Lechner, M., Pon, Z., Brink, D., Abbasy, I., Jaafar, M., 2009. Case study: Successful application of a novel conformance treatment in an extended reach horizontal well in the Al Shaheen field, offshore Qatar, in: SPE Offshore Europe Oil \& Gas Conference \& Exhibition, Aberdeen, UK.

Qian, K., Zhang, Y., 2014. Bilinear model predictive control of plasma keyhole pipe welding process. Journal of Manufacturing Science and Engineering, Transactions of the ASME 136, 031002-1 - 031002-10.

Rade, L., Westergren, B., 1999. Mathematics Handbook for Science and Engineering. 4th ed., Springer, Berlin - Tokyo.

Rugh, W.J., 1981. Nonlinear System Theory: The Volterra/Wiener Approach. Johns Jopkins University Press, Baltimore, MD.

Schwarz, H., Dorissen, H.T., 1989. System identification of bilinear systems via realization theory and its application. Control, Theory and Advanced Technology 5, 137-155.

Short, J.A., 1993. Introduction to Directional and Horizontal Drilling. PennWell Publishing Company, OK.

van de Wouw, N., Monsieurs, F.H.A., Detournay, E., 2016. Dynamic state-feedback control of nonlinear three-dimensional directional drilling systems. IFAC-PapersOnLine 49, 85-90.

Yinghui, L., Yinao, S., 2000. Automatic inclination controller: A new inclination controlling tool for rotary drilling, in: IADC/SPE Drilling Conference, New Orleans, LA.

555 Yonezawa, T., Cargill, E., Gaynor, T., Hardin, J., Hay, R., Ikeda, A., Kiyosawa, Y., 2002. Robotic controlled drilling: A new rotary steerable drilling system for the oil and gas industry, in: IADC/SPE Drilling Conference, Texas, USA. 
2018-10-25

\section{Bilinear modelling, control and stability of directional drilling}

Inyang, Isonguyo J.

Elsevier

Isonguyo J. Inyang and James F. Whidborne. (2019) Bilinear modelling, control and stability of directional drilling. Control Engineering Practice, Volume 82, January 2019, pp. 161-172

https://doi.org/10.1016/j.conengprac.2018.10.008

Downloaded from Cranfield Library Services E-Repository 